\title{
Dynamical features of higher-order correlation events: impact on cortical cells
}

\author{
Andrea Benucci · Paul F. M. J. Verschure • \\ Peter König
}

Received: 27 April 2006/ Accepted: 10 October 2006/Published online: 25 November 2006

(C) Springer Science+Business Media B.V. 2006

\begin{abstract}
Cortical neurons receive signals from thousands of other neurons. The statistical properties of the input spike trains substantially shape the output response properties of each neuron. Experimental and theoretical investigations have mostly focused on the second order statistical features of the input spike trains (mean firing rates and pairwise correlations). Little is known of how higher order correlations affect the integration and firing behavior of a cell independently of the second order statistics. To address this issue, we simulated the dynamics of a population of 5000 neurons, controlling both their second order and higher-order correlation properties to reflect physiological data. We then used these ensemble dynamics as the input stage to morphologically reconstructed cortical cells (layer 5 pyramidal, layer 4 spiny stellate cell), and to an integrate and fire neuron. Our results show that changes done solely to the higher-order correlation properties of the network's dynamics significantly affect the response properties of a target neuron, both
\end{abstract}

\footnotetext{
A. Benucci $(\bowtie)$

Smith-Kettlewell Eye Research Institute, 2318 Fillmore St., San Francisco, CA 94115, USA

e-mail: andrea@ski.org

P. F. M. J. Verschure

Institució Catalana de Recerca i Estudis Avançats

(ICREA), Technology Department, University Pompeu

Fabra, Passeig de Circumval-lacio 8, 08003 Barcelona, Spain

e-mail: paul.verschure@upf.edu

P. König

Institut für Kognitionswissenschaft - Neurobiopsychologie,

Universität Osnabrück, Albrechtstraße 28, 49069

Osnabrück, Germany

e-mail: pkoenig@uos.de
}

in terms of output rate and spike timing. Moreover, the neuronal morphology and voltage dependent mechanisms of the target neuron considerably modulate the quantitative aspects of these effects. Finally, we show how these results affect sparseness of neuronal representations, tuning properties, and feature selectivity of cortical cells.

Keywords Correlations - Unitary events .

Single-cell model $\cdot$ Sparseness $\cdot$ Neuronal selectivity

\section{Introduction}

The statistical properties of the inputs to a target neuron have a great impact on its response properties, such as the firing rate, spike timing variability, and reliability (Salinas and Sejnowski 2001). Nevertheless, the statistical features of the inputs on a millisecond time scale are currently not known. Higher-order correlation events (also called unitary events) define time epochs in the activity of an ensemble of cells, characterized by a conspicuous quasi-synchronous spiking of a large fraction of neurons (Grün et al. 2001; Abeles 1991; Bothé et al. 2000). The large majority of experimental and modeling studies are classically concerned with second order correlation properties of the dynamics (firing rates, coefficient of variation - CV, and pairwise correlations), neglecting the variability of the higher-order correlation terms (Salinas and Sejnowski 2000; Bernander et al. 1994; Feng and Brown 2000). Here we use a computational approach to show that cortical neurons are extremely sensitive detectors of the higher-order correlation properties of 
their inputs, thus suggesting that unitary events are deeply involved in neuronal computation and in the mechanisms used by the neurons to encode and transmit information.

To address the question of the impact of higher-order correlation event on the dynamics of cortical neurons, we used the following quantitative approach: we generated an ensemble of spike trains mimicking the dynamics of a large group of neurons. A key feature of the algorithm is the possibility to produce changes in the structure of the higher-order correlation events, while keeping firing rates, $\mathrm{CV}$, and pairwise correlations fixed. We then introduced detailed compartmental models of a cortical Layer 5 pyramidal neuron, a Layer 4 spiny stellate cell and a single compartment, conductance based model of an integrate and fire neuron. The effects of the spike trains were tested on these modeled cells. This allowed us to selectively evaluate the impact of unitary events on the output response properties of the modeled neurons, excluding possible contributions due to changes in the second order correlation properties.

We performed analyses in the time and frequency domain, in the supra- as well as in the sub-threshold domain. Significant changes are observed in each of these scenarios.

In a sequence of controls aimed at studying the scope of the results with respect to modeling parameters, we modified the neuronal morphologies and voltage dependent mechanisms. We showed that the qualitative features of the results are robust for a wide choice of simulation parameters. Moreover, quantitative and qualitative differences emerged whether or not the neuronal morphology was explicitly implemented.

Finally, we highlighted effects of unitary events on population sparseness (Willmore and Tolhurst 2001) and effects on orientation selectivity and tuning properties of single neurons.

\section{Method}

In the following section we describe the algorithm used to selectively manipulate the higher-order correlation properties of the network dynamics, while keeping the second order parameters constant, i.e. the mean firing rate, the coefficient of variation in the single-cell spiking activity, and pairwise correlation strength between any two given spike trains (see next section for the mathematical definitions of these quantities). We did not use a formal analytical approach. Instead,
Fig. 1 Schematic representation of the algorithm used to generate spike correlations. (a) Top panel: For simplicity, a set of 3 spike trains is considered; each train has a duration $T$ and time resolution $\Delta T_{\text {bin. }} .7$ spikes are assigned to each spike train, as schematized with light gray and full circles in the central-left part of the Figure. The 4 light gray spikes are assigned to 4 time epochs randomly chosen from a group of 6 (E1...E6). Each epoch is centered on a time-marker (Sd1...Sd6, see main text). The epoch duration is $\Delta T_{\mathrm{E}}$. Each of the light gray spikes is assigned to a given bin within an epoch according to a Gaussian distribution $\left(\operatorname{PDF}\left(S_{\mathrm{A}}\right)\right.$ central part of the Figure). For any given pair of cells 2 spikes occur in the same epochs, thus pairwise correlations are created (E3 and E4 for cell 1 and 3; E1 and E2 for cell 1 and 2 and so on). The not assigned spikes (full circles) are randomly distributed in between the empty bins (not shown in the Figure), with the constraint of refractoriness, and inter-spike-intervals (ISI) compatible with a $C V \cong 1$ (see main text). Bottom panel: Same procedure is applied for the 3 cells, but starting with a different set of spikes and epochs: 3 spikes are assigned to only four epochs. For each pair of cells there are 2 spikes occurring in the same epochs (pairwise correlation strength as before), but it is not possible to avoid vertical alignment of 3 spikes for at least one of the 4 epochs (the second epoch in the schematic), thus a triplet is created. (b) $P(n)$ distributions for three sets of $S_{\mathrm{A}}$ and $N_{\mathrm{A}}$ : 29-36-39 Hz and $84-133-153 \mathrm{~Hz}$, respectively. The mean number of correlated spikes for each pair of trains is given by $\langle n\rangle=\frac{S_{\mathrm{A}}^{2}}{N_{\mathrm{E}}} \cong 9 \mathrm{~Hz}$ for each of the three sets. This choice of parameters is optimized for a pairwise correlation strength of 0.1 and a mean firing rate of $85 \mathrm{~Hz}$, i.e. 8.5 correlated spikes per second. Indeed, the bin $n=9$ is associated to a peak probability ranging around 0.18 . Thus, at least 28000 spike trains must be generated to obtain 5000 trains with the required pairwise correlation properties (see Section 2.2)

we created a numerical algorithm, which is based on a probabilistic method.

\section{Description of the algorithm}

The goal of the algorithm is to produce a large ensemble of spike trains, more specifically produce an ensemble of 5000 spike trains, lasting $T$ seconds each $(T=10 \mathrm{~s})$. Spikes are not assigned randomly, so that temporal correlations between the spikes are introduced. For the sake of clarity, we will use the schematic representation of Fig. 1a to exemplify the concepts describing the algorithm.

- Within the overall time window of analysis $T$, time epochs $\left(E_{1} \ldots E_{6}\right.$, top panel) are pre-selected for all the spike trains in the ensemble, during which the correlated spiking activity can occur. The time window is subdivided in small time bins of width $\Delta T_{\text {bin }}=0.1 \mathrm{~ms}$, which represents the time resolution of the system. The frequency of the time epochs $\left(f_{\mathrm{E}}=\frac{N_{\mathrm{E}}}{T}\right.$, with $N_{\mathrm{E}}$ the total number of epochs in the interval $T$ ) can be 84, 133, and $153 \mathrm{~Hz}$ (see also the Result section); for simplicity, 

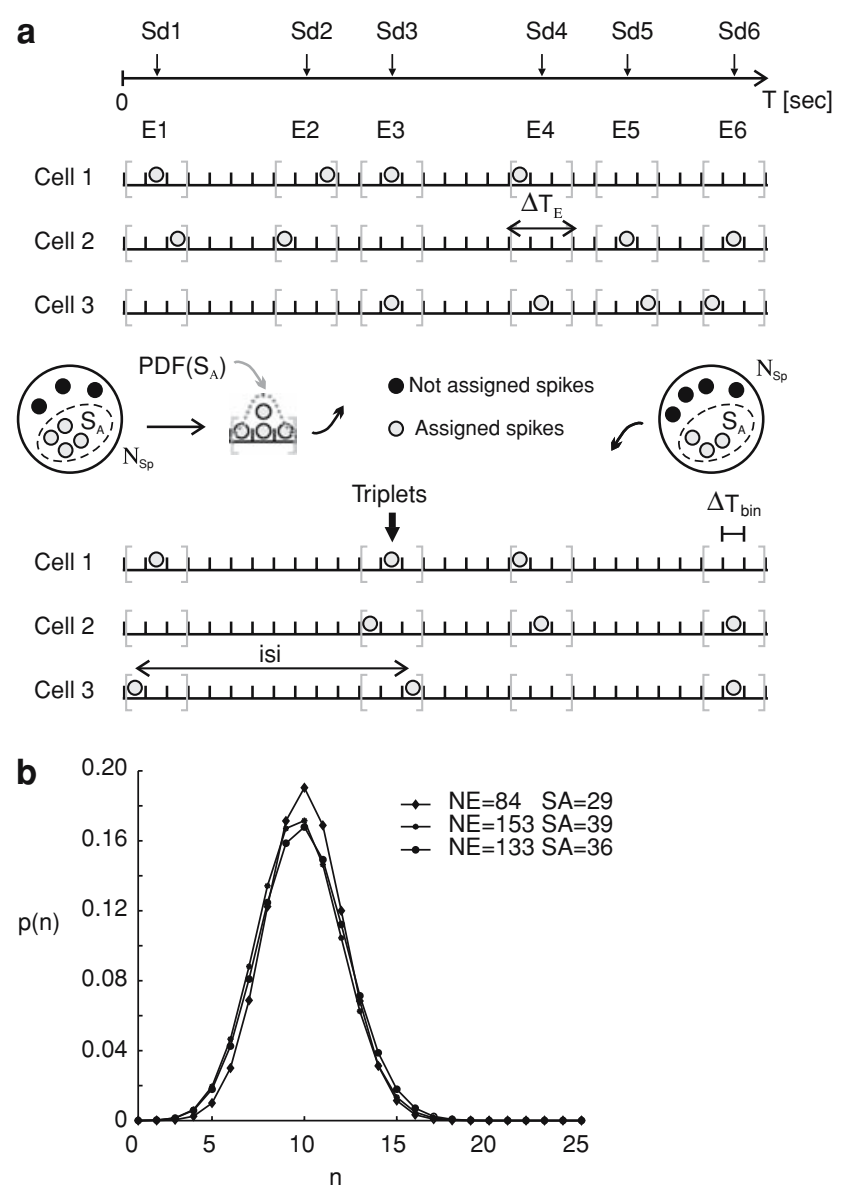

only an ensemble with 3 spike trains is considered in the example of Fig. 1a. The epochs are time intervals, of duration $\Delta T_{\mathrm{E}}$ (lasting $10 \mathrm{~ms}$ ), centered on specific points in time, whose distribution is a controlled (independent) parameter of the algorithm. We refer to these points as the "seeds" of the dynamics $(\mathrm{Sd} 1 \ldots \mathrm{Sd} 6$; the occurrence of a seed is indicated by short vertical arrows pointing to different time locations within the window $T$, top part of Fig. 1a). By definition, the frequency of the seeds is the same as the frequency of the epochs: $f_{\mathrm{E}}=f_{\mathrm{Sd}}=\frac{N_{\mathrm{Sd}}}{T}$, with $N_{\mathrm{Sd}}$ the total number of seeds $(6$ in the schematic of Fig. 1a, top panel). The interseeds intervals, the separation in time between the seeds $\left(\mathrm{IS}_{\mathrm{d}} \mathrm{I}_{j}=t_{\mathrm{Sd} j+1}-t_{\mathrm{Sd} j}\right)$, and accordingly the times of occurrence of the seeds $\left(t \mathrm{Sd}_{j}\right)$, are set by a random number generator according to an exponential distributions $\operatorname{PDF}\left(\operatorname{IS}_{\mathrm{d}} \mathrm{I}_{j}, \mu\right)=\frac{1}{\mu} \mathrm{e}^{-\frac{\mathrm{IS}_{\mathrm{d}} \mathrm{d}_{j}}{\mu}}$, with $\mu=\frac{1}{f_{\mathrm{Sd}}}$ s. To emphasize the generality of the algorithm, a different distribution (Poisson or Gamma of any order) would allow the creation of correlations with or without overall oscillatory activity (Gray and Viana di Prisco 1997; Engel et al. 1990), see Fig. 2c-e.
- Every spike train has a fixed mean firing rate $f_{\mathrm{Sp}}=\frac{N_{\mathrm{Sp}}}{T}$, with $N_{\mathrm{Sp}}$ the total number of spikes in a time window $T$ (the total number of spikes for each spike train in the central left and right part of Fig. 1a, is $N_{\mathrm{Sp}}=7$, and it is represented as a group of small full, as well as open, circles within a larger open circle). A subset of spikes, $S_{\mathrm{A}}$ (4 small open circles surrounded by a dotted gray oval; note that for convenience, only these spikes are shown in the spike trains), is assigned to a randomly chosen sub group, $n_{\mathrm{E}}$, of epochs (the frequency of occurrence of $n_{\mathrm{E}}$ can be $29 \mathrm{~Hz}, 36 \mathrm{~Hz}$, and $39 \mathrm{~Hz}$ ). Each epoch $n_{\mathrm{E}}$ receives only one spike, thus $\left|n_{\mathrm{E}}\right|=|S A|\left(n_{\mathrm{E}}=4\right.$ in Fig. 1a, top panel; note that each random choice is different for different spike trains, but the total number of selected epochs is always 4: E1, E2, E3, E4 for cell1, E1, E2, E5, E6 for cell2 and so on). Each assigned spike $S_{\mathrm{A}}$, is allocated in a different time-position within its matching epoch, according to a normal distribution (labeled as $\operatorname{PDF}\left(t_{\mathrm{SA}}\right)=\frac{1}{\sigma \sqrt{2 \pi}} e^{-\frac{\left(t_{\mathrm{SA}}-t_{\mathrm{E}}\right)^{2}}{2 \sigma^{2}}}$, with $t_{\mathrm{E}}$ the time of occurrence of the epoch and $\sigma=\frac{\Delta T_{\mathrm{E}}}{2}$, dotted gray line). For example in Fig. 1a, according to the normal distribution, the 4 assigned spikes of cell 1 should preferentially cluster in the center of the corresponding epochs (E1, E2, E3, and E4). Thus one spike is assigned to the central time bin of epoch E1 and E3, one spike is assigned to the left time bin of E4 and the last spike to the right time bin of E2. The same is done for cell 2 and 3, but with different selections of epochs. The specific order in which assigned spikes of $\operatorname{PDF}\left(t_{\mathrm{SA}}\right)$ are associated to time-bins within epochs is not relevant: assignments are done randomly in the algorithm.

- Due to combinatorial arguments, some epochs must be selected multiple times for different cells. In general, the total number of ways to select $S_{\mathrm{A}}$ epochs from a pool of $N_{\mathrm{E}}$ is given by the combinatorial factor $C_{N_{\mathrm{E}}, S_{\mathrm{A}}}=\frac{N_{\mathrm{E}} !}{S_{\mathrm{A}} !\left(N_{\mathrm{E}}-S_{\mathrm{A}}\right) !}\left(C_{6,4}=15\right.$, in the example of Fig. 1a, top panel). Moreover, for any two given cells and for any choice of epochs in between the $C_{N_{\mathrm{E}}, S_{\mathrm{A}}}$ available, the number of correlated spikes $n$ follows the distribution function $p(n)=\frac{C_{\left(S_{\mathrm{A}}, n\right)} C_{\left(N_{\mathrm{E}}-S_{\mathrm{A}}, S_{\mathrm{A}}-n\right)}}{C_{\left(N_{\mathrm{E}}, S_{\mathrm{A}}\right)}}$. From this equation, the average number of correlated spikes is $\langle n\rangle=\frac{S_{\mathrm{A}}^{2}}{N_{\mathrm{E}}}$.

- According to this strategy, pairwise correlations are produced between spike trains. The duration $\Delta T_{E}$ determines the temporal precision of the correlations, and the parameter $\langle n\rangle$ specifies the average number of common spikes between pairs of cells, 
Fig. 2 Examples of different dynamics produced by the algorithm. (a) Left, raster plot and corresponding population PSTH, for a sub-sample of 100 cells firing at $40 \mathrm{~Hz}$. The crosscorrelograms, normalized for the peak amplitude, are shown in the right panels. (b) Uncorrelated spike trains are shown with non-stationary (increasing) firing rate during the first $200 \mathrm{~ms}$. Note that only stationary cases are considered in this study (c) Oscillatory activity in the gamma frequency band $(50 \mathrm{~Hz})$ obtained by using a Poisson PDF for the Inter Unitary Events Intervals, see main text. (d) By using a gamma function, and by changing the PDF associated to the unitary-events-related spikes (see text), it is possible to produce correlated, non oscillatory activity, with sharp satellite peaks at roughly $15 \mathrm{~ms}$ time lags, as shown in the cross-correlogram. (e) Changing the same set of parameters, "smoother", secondary peaks can be produced, with wider central peaks, mimicking strong correlations with lower temporal precision

i.e. the mean pairwise correlation strength. Though, according to the distribution $p(n)$, a significant fraction of spike-train pairs produced by the algorithm will not have the desired pairwise correlation strength (i.e. all of those that are not in the $\langle n\rangle$-bin of the distribution). To overcome this problem, we introduced a selection criterion that allowed us to narrow down only trains with the required correlation properties, see Section 2.2.

- The key property of the algorithm is that it is possible to keep $\langle n\rangle$ constant (pairwise correlations), while decreasing the number of seeds (and epochs $N_{\mathrm{E}}$ as well) by changing the value of $S_{\mathrm{A}}$ accordingly. This is exemplified in the bottom panel of Fig. 1a: decreasing the frequency of the seeds $f_{\mathrm{Sd}}$, while keeping the firing rate and pairwise correlation strength constant, produces an increase in the higher-order correlations between the spike trains: triplets are necessarily formed in the example of Fig. 1a, bottom panel, but not in the top panel, where, by exploiting all the combinatorial possibilities $C_{N_{\mathrm{E}}, S_{\mathrm{A}}}$, it is possible to create only correlated spike-pairs.

- The remaining spikes, $N_{\mathrm{Sp}}-S_{\mathrm{A}}$ (3 and 4 full small circles in the central left and right part of Fig. 1a, respectively) are distributed randomly in time according to a Poisson process (not shown in the Fig. 1a), so that the coefficient of variation $\left(\mathrm{CV}=\frac{\left(\operatorname{Var}\left[T_{\mathrm{th}}\right]\right)^{1 / 2}}{\left\langle T_{\mathrm{th}}\right\rangle}\right.$, with $T_{\text {th }}$ the time between two consecutive spikes) ranges around 1 (Dean 1981), i.e. the inter-spike-interval $\left(\operatorname{ISI}_{k}=\left|t_{k}-t_{k}-1\right|\right.$; $\left.k=2, \ldots, N_{s p}\right)$ distribution is well approximated by an exponential function. The only constraint for the unassigned spikes is refractoriness $\left(\mathrm{R}=\min \left[\left(t_{k}-\right.\right.\right.$ $\left.\left.t_{k}-1\right) ; k=2, \ldots, N_{\mathrm{sp}}\right]$ the minimum time between two consecutive spikes) does not assume values smaller than $2 \mathrm{~ms}$. These spikes can also occur
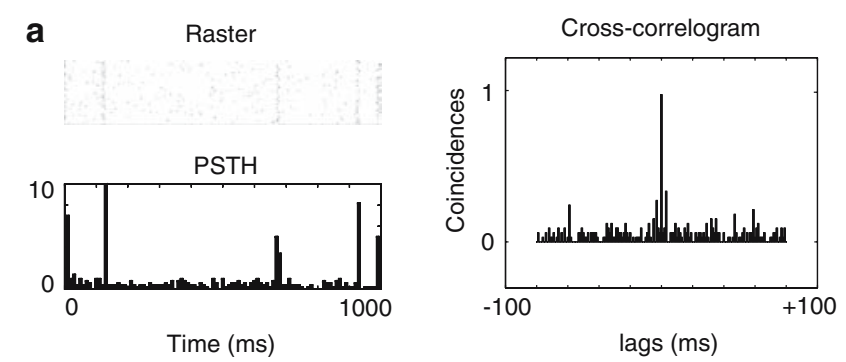

b

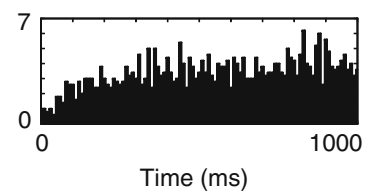

C

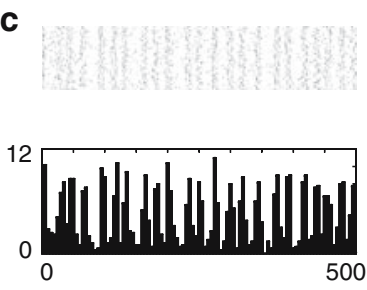

Time (ms)

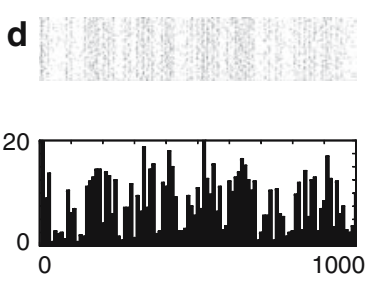

Time (ms)

e

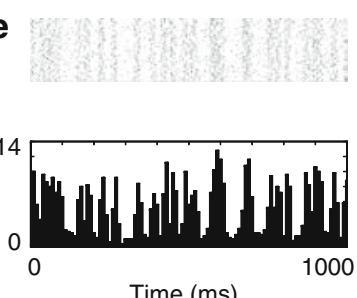

Time (ms)

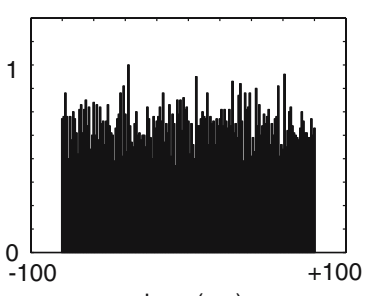

lags (ms)

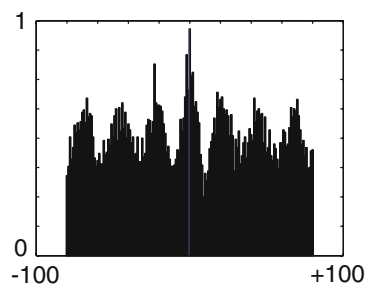

lags (ms)

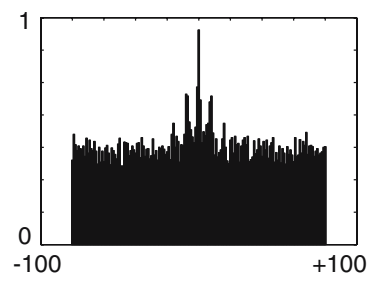

lags (ms)

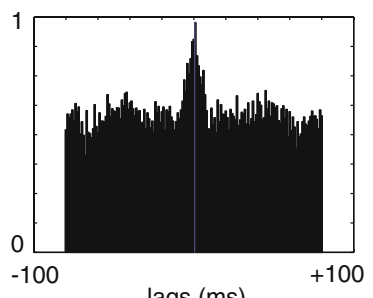

lags (ms)

within epochs, but since they are randomly distributed, the additional correlations they might introduce can only occur by chance, without promoting any specific epoch or seed.

Thus, by keeping the parameters $\langle n\rangle, \mathrm{CV}, f_{\mathrm{Sp}}$ (i.e. the second order correlation properties) constant, while varying $N_{\mathrm{E}}$ and $S_{\mathrm{A}}$, it is possible to selectively modulate the higher-order correlation properties of the population dynamics. The parameters $\Delta T_{\mathrm{E}}$ and $\operatorname{PDF}\left(t_{S_{\mathrm{A}}}\right)$ modulate the temporal precision of the correlations. In the simulations described in the following, 
we used three sets of $\left(N_{\mathrm{E}}, S_{\mathrm{A}}\right)$-frequency of occurrence (and accordingly three $p(n)$ distributions, Fig. 1b): $84 \mathrm{~Hz}, 133 \mathrm{~Hz}$, and $153 \mathrm{~Hz}$, and $29 \mathrm{~Hz}, 36 \mathrm{~Hz}$, and $39 \mathrm{~Hz}$, for $N_{\mathrm{E}}$ and $S_{\mathrm{A}}$ respectively, with $\langle n\rangle=10,9.7$, and 9.9, respectively. Some examples of correlated spiking dynamics generated by the algorithm are shown in Fig. 2.

\section{Probabilistic features of the algorithm}

The spike generation algorithm is probabilistic, thus it is necessary to check whether the resulting spike patterns conform to our requirements. Indeed, as explained above, the precise time of occurrence of the seeds is determined by a random number generator; the same is true for the time of occurrence of the spikes within each time epoch, and for the time of occurrence of the spikes that are not assigned to any time epoch, according to the refractoriness constraint. As a result, the number of correlated spikes between pairs of spike trains is described by the distribution function $p(n)$.

To verify that the generated spike trains have the expected pairwise correlation strength, mean firing rate and $\mathrm{CV}$, we used an "experimental" approach, i.e. we treated the synthetic data as standard single unit recordings and performed commonly used statistical analyses to characterize, a-posteriori, the dynamical properties of the spike trains: First, we verified that, within the overall time window $T$, the mean firing rates and CV had the expected values. Second, we computed cross-correlation functions between pairs of spike trains and derived average cross-correlograms. Given that the required number of spike trains is 5000 , it is not computationally convenient to analyze crosscorrelagrams for each and every pair of spike trains. Indeed, the combinatorial possibilities scale like $\frac{n_{\text {trains }}^{2}}{2}$, with $n_{\text {trains }}$ the size of the ensemble: given 5000 spike trains, an order of $10^{7}$ cross-correlograms should be computed. Thus we used the following approach: Suppose the algorithm has generated $s_{1}, \ldots, s_{n}$ spike trains (with $n \approx 10^{4}$ ). We took a permutation of the original sequence $p_{1}^{1} \ldots p_{n}^{1}=\mathrm{P}^{1}\left(s_{1} \ldots s_{n}\right)$ and used it to compute $n$ cross-correlograms between all the pairs $\left(s_{1}, p_{1}^{1}\right),\left(s_{2}, p_{2}^{1}\right),\left(s_{3}, p_{3}^{1}\right), \ldots,\left(s_{n}, p_{n}^{1}\right)$. To speed up the computation, only zero-lag correlations have been considered, thus the only parameter of interest is the value of the cross-correlogram at zero-lag. We repeated the analysis for different permutations, $\mathrm{P}^{j}\left(s_{1} \ldots s_{n}\right), j=1 \ldots$ $w$. If $\overline{p k}$ is the required peak value of the cross-correlogram, (i.e. the value associated to the desired pairwise correlation strength) then the average peak value for the $i^{\text {th }}$ spike train is $\left\langle p k_{i}\right\rangle=\frac{1}{w} \sum_{j=1} \alpha_{i}^{j} \times \overline{p k}$, where the $\alpha$-values are the differences between the computed peak value and the expected one, $\overline{p k}$. Thus, a distribution of $\alpha$-values, $\operatorname{pdf}_{i}\left(\alpha^{j}\right)$, is associated to each spike train. The spike trains used in the simulations are those whose distributions have a mean close to one, and a narrow width (standard deviation). To quantify whether the mean was close to one, we used a Student ttest with a $95 \%$ confidence interval. For the width we used a tradeoff: very narrow widths imply too few surviving spike trains; wider widths imply large variability in the pairwise correlation strength. $12 \%$ variability in the expected value of the correlations (i.e. $~ 1$ correlated spike per second, at $85 \mathrm{~Hz}$ for pairwise correlation strength of 0.1 ) is what we used. This criterion is justified by the observation that changes in pairwise correlation strength within this range of variability do not affect the response properties of the modeled target neurons (as described in Section3). In other words, if we impose a narrower tuning width on the $\alpha$-distribution (average variability lower than 1 correlated spike per second), the first-order response properties of the modeled target neuron (mean firing rate and $\mathrm{CV}$ ) are indistinguishable from what observed with a $12 \%$ criteria.

From a computational point of view, with $w=200$ permutations, only $5-10 \%$ of the spike trains had the required pairwise correlation properties and could be used as inputs to the modeled cells. Thus, to select 5000 input spike trains, an initial pool of $50-100 \times 10^{3}$ spike trains must be generated.

Finally, we checked for stationarity: given the probabilistic nature of the algorithm and the limited duration of the spike trains, the distribution of the seeds was not always "homogeneous" in time. Thus, occasionally, periods lasting a few hundreds of milliseconds with increased mean firing rates were formed. To select only sets of spike trains with optimal stationary properties, we used a sliding time window of $300 \mathrm{~ms}$ duration, with overlapping time intervals of $100 \mathrm{~ms}$, and computed average firing rates, coefficients of variation, and pairwise correlation strengths. Only those sets that followed the required criteria, as explained above, were used for the simulations. This allowed the clear separation of three populations of spike trains, which differed from each other solely in the higher-order correlation properties, while having identical pairwise correlation strength, $\mathrm{CV}$, and mean firing rate. Qualitatively, the first ensemble is characterized by a distribution of spikes that is very sparse in time, with a "low" degree of higherorder correlation events; the third population has a "very high" degree of higher-order correlation event, and the second one is sitting in between. 
Limitations of the algorithm

As pointed out in the previous section, due to the probabilistic nature of the algorithm, we could reliably separate no more than three groups of spike trains with parametrically controlled second order and higherorder correlation values. Contributing to this problem there is a second issue related to the frequency saturation for the unitary events: in the limiting case of No. Seeds $=\frac{T}{\Delta t_{\text {bin }}}$ (i.e. the maximum seeds number for a given time resolution) all the time bins are equally considered as possible seeds for the creation of highest-order correlation events. In this limiting case, the pairwise correlations drop to zero since the probability of common firing between spike trains is "diluted" all over the time bins and the instantaneous population activity undergoes a random walk dynamic (data not shown). Accordingly, in the simulation, after a maximum frequency of the unitary events $(250 \mathrm{~Hz})$, a "saturation point" is reached such that the pairwise correlation strength cannot be held constant, and it starts to decrease (note that this is not a theoretical limit, but a numerical limit related to the properties of the algorithm used). The results shown in the following are obtained with a choice of parameters far from the limiting case and the strength of the pairwise correlations has been held constant for the different frequency-values of the unitary events.

\section{Orientation properties of the excitatory} and inhibitory inputs

The selected ensembles of spike trains have been generated to provide inputs to detailed compartmental models of cortical cells. Cortical cells receive input from neurons having different stimulus preferences, thus different input spike trains have different statistical properties. To take into account this variability, we simulated optimal and sub-optimal virtual stimulation: we divided the 5000 input spike trains in 36 subgroups of different sizes, representing subsets of inputs coming from cells having different tuning properties from the target cell, following known connectivity data (Kisvarday et al. 1997). 4000 spike trains were firing according to a standard orientation tuning curve: the largest group of cells (400 cells) firing at $85 \mathrm{~Hz}$ and the smallest group (90 cells, representing the orthogonal orientation) at $4 \mathrm{~Hz}$. The remaining 1000 spike trains were firing according to a broader tuning curve; these inputs have been used to activate the GABA A/B synapses, while the former 4000 were targeting AMPA synapses (see Section2.5). This provided the cell with a purely feed forward mechanism for the orientation tuning, and allowed us to simulate optimal and sub-optimal stimulation to the modeled neurons. In the optimal-stimulation condition the largest of the 36 input groups of cells (400 cells) was firing at the highest rate $(85 \mathrm{~Hz})$ and the smallest group (90 cells) at the lowest rate, $4 \mathrm{~Hz}$. The groups in between were firing at intermediate rates, according to a standard tuning curve (Ferster and Miller 2000), see also Section 3.2.3 and Appendix. In the sub-optimal (or orthogonal) condition the largest input group was firing at $4 \mathrm{~Hz}$, and the smallest group at $85 \mathrm{~Hz}$. The distribution of the AMPA synapses, located in the spine heads, was uniform along the dendritic tree, while the GABA synapses were restricted to the basal dendrites and the initial part of the apical tuft. The synaptic parameters are reported in the Appendix.

\section{Description of the modeled cells}

Using the NEURON simulation environment (Hines et al. 2000), we implemented three different modelcells, meant as receivers of the population's activity: (1) a detailed compartmental model of a layer five pyramidal cell, (2) a layer four spiny stellate cell and (3) a conductance based Integrate and Fire neuron. We chose these three models because they allowed us to test the generality of the results when the cell morphology is considered (layer five pyramidal and layer four spiny stellate vs. a single geometrical point neuron), as well as to make comparisons between different common cellular morphologies (layer five pyramidal vs. layer four spiny stellate). All the simulations had a time resolution of $1 \mathrm{~ms}$ and single trial duration, $T$, of $10 \mathrm{~s}$. The details of the parameters used are described in the Appendix. The synaptic parameters were chosen to match the variability (CV), and firing rates of the output spike trains, with those of the inputs, i.e. the balance of the cell (Salinas and Sejnowski 2000) has been set so that the cell responded with the same rate and spike variability as the input network. Moreover, several voltage dependent mechanisms and ionic channels have been used to simulate dendritic nonlinearities (see Appendix). In a sequence of controls (see Section4) we tested the robustness of the results with respect to changes in the simulation parameters.

\section{Results}

We used the three previously described sets of spike trains as input stages to the modeled neurons. Since the input-sets differ from each other only in their highest-order correlation properties, any effect on the 
output responses depends uniquely on the variability of highest-order correlations. In the sub-threshold domain, we investigated the statistical properties of the membrane potentials, both in the time and frequency domain. In the supra-threshold domain we looked at the mean firing rates, temporal variability (coefficient of variation), and spike timing precision. Finally, we ran a sequence of controls changing synaptic parameters, neuronal electrotonic properties, and morphological features.

\section{Presynaptic dynamics}

In order to understand the output response properties of the modeled neurons, it is necessary to quantify the population dynamics at the input stage at fine time scales. As a measure of the instantaneous ensemble activity, we computed population PSTHs (Peri-Stimulus Time Histograms) derived from the whole ensemble of input spike trains, for the three different frequencies of the unitary events:
84, 133, $153 \mathrm{~Hz}$ respectively, Fig. 3, left column. Pairwise correlation strength, coefficient of variation, and mean firing rate of the spike trains have been kept constant: correlation strength $\mathrm{c}=0.1, \mathrm{CV}=1$, $f=85.4 \mathrm{~Hz}$. The histograms of the population PSTHs are shown in Fig. 3, right column. The major difference between the three cases is a change in the bimodality: the higher the frequency of the unitary events, the stronger is the histograms' bimodality. This effect has an easy qualitative explanation: an increased frequency of the unitary events is related to an increased number of peaks in the population PSTHs. The amplitude of the peaks decreases with increasing frequencies, but the fraction of the total number of spikes associated to the unitary events increases. It follows that a shift is produced in the relative proportion of spikes assigned randomly vs. those that are associated to unitary events, with the result of skewing the histograms. Since the membrane potential gets, on average, closer to threshold with increasing rates of unitary events, it would be
Fig. 3 Effects of changes in the frequency of the unitary events on the presynaptic dynamics. (a) Left, population PSTH for 300 cells spiking at $85 \mathrm{~Hz}$, with pairwise correlation strength of $0.08, \mathrm{CV}=1$, and $81 \mathrm{~Hz}$ for the frequency of the unitary events. On the right side, the histogram of the population PSTH is characterized by a sharp primary peak, followed by a smaller and smoother secondary peak (the unitary events frequency, 'Sd', is reported in the insets). (b) Increasing the frequency of the seeds to $133 \mathrm{~Hz}$ causes an increase in the amplitude of the secondary peak. This effect is even more pronounced at $153 \mathrm{~Hz}$, (c). In all three cases the mean firing rate, $\mathrm{CV}$ and pairwise correlation strength are kept constant and the observed changes in the shape of the histograms are solely due to the variations in the higherorder correlation structure
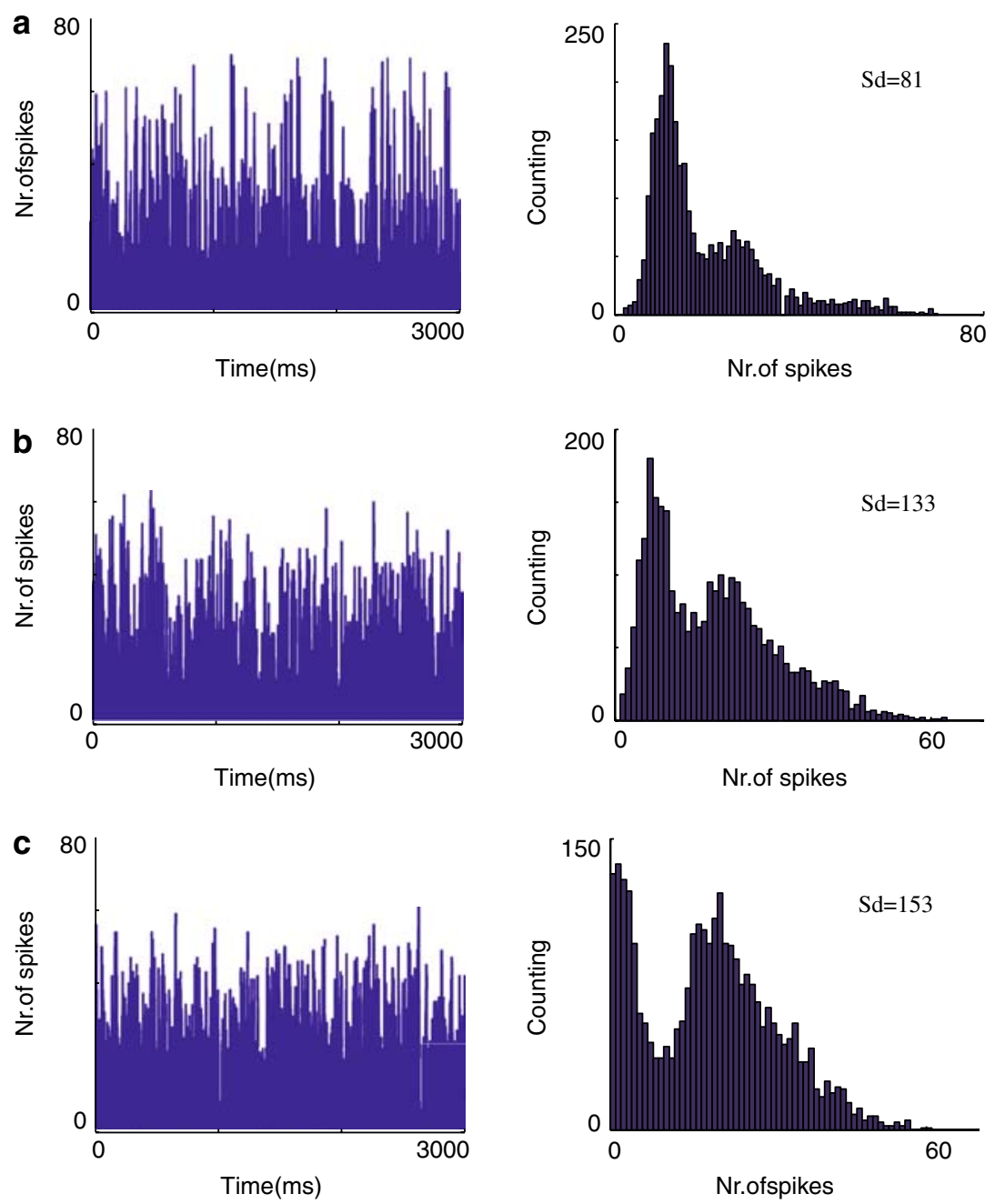
intuitive to expect an increase in the output firing rate as well. We will show later that surprisingly, this intuition does not always hold true.

Power-spectra analysis of the input population PSTHs, Fig. 4a, reveals an increased power only in the $10-20 \mathrm{~Hz}$ band when the frequency of the unitary increases from $81 \mathrm{~Hz}$ to $153 \mathrm{~Hz}$, as before. The frequency content of the inputs could be relevant to trigger stochastic resonance phenomena in the postsynaptic membrane potential, as shown in Rudolph and Destexhe (2001), and thus affecting the output rates. Nevertheless, the observed increase is significant only for pairwise correlation strengths above 0.1 , outside the range considered in the present study.

\section{Post-synaptic dynamics}

Unless otherwise specified, the following results have been obtained by using a detailed morphologically reconstructed layer five pyramidal cell with passive dendrites and Hodgkin and Huxley spiking mechanisms in the soma (see Appendix).

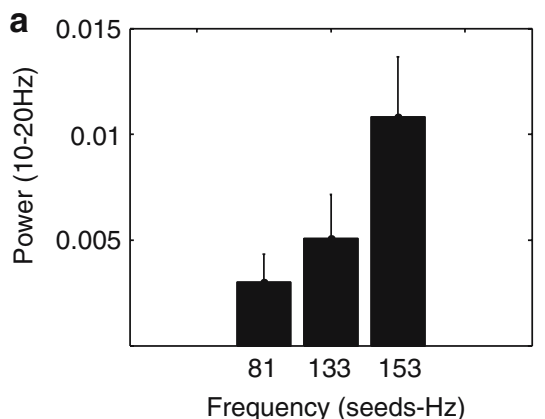

C

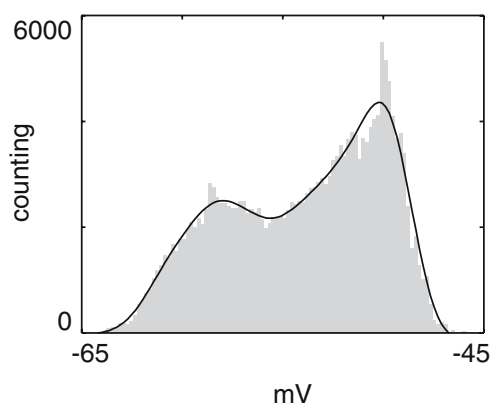

Fig. 4 (a) Power spectra analysis of three population PSTHs obtained with different frequencies of unitary events $(81,133$, and $153 \mathrm{~Hz}$, respectively) and with an average correlation strenght of 0.25 (see Method section). The difference in power, observed only in the 10-20 Hz frequency band, as shown in this panel, disappears for pairwise correlation strength with a relative modulation amplitude lower than 0.1. Arbitrary units of power. (b) Intracellular recording from the layer 5 pyramidal cell, during optimal stimulation and 0.1 pairwise correlation strength of the inputs (same in $\mathrm{c}$ and $\mathrm{d}$ ). The cell is responding at $85 \mathrm{~Hz}$

\section{Subthreshold properties}

A typical trace of an intracellular somatic recording is shown in Fig. 4b (dark gray trace). After removing the spikes from the voltage traces (Azouz and Gray 2000), we computed the histogram of the membrane potential $\left(V_{\mathrm{m}}\right)$. The effect of changing the frequency of the presynaptic unitary events is mainly related to variations in the skewness of the histograms (Fig. 4c-d). When the frequency of unitary events increases, the amplitude of the more depolarized peak increases relative to the more hyperpolarized one. To quantify this effect we used a dimensionless index (A1/A2-index) defined as the ratio between the amplitude of the two peaks. As shown in Fig. 5a, the index is a monotonically decreasing function of the frequency of the unitary events. The interpretation of the phenomenon is again intuitive. More unitary events imply more peaks in the presynaptic activity profiles (population PSTHs); this in turn would "force" the membrane potential more often toward depolarized states. Since the area of the peaks is proportional to the time spent
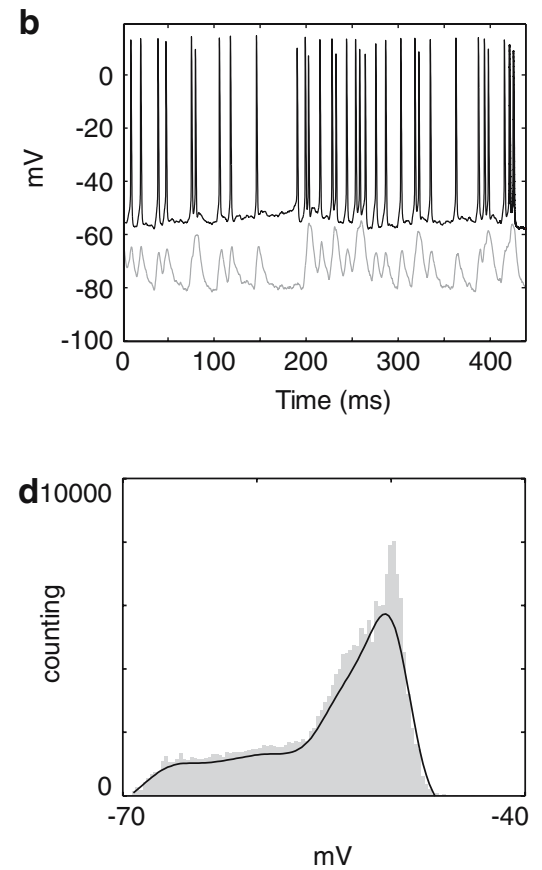

(consistently with the maximum input firing rate), with a coefficient of variation $\mathrm{CV}=1$. The bottom light gray trace represents the same somatic recording with an additional 6 nAmp I-clamp to stop the cell from firing. (c-d) When the frequency of the unitary events increases from $133 \mathrm{~Hz}$ to $153 \mathrm{~Hz}$ (panel c and d, respectively), without any change in the second order correlation properties, the shape of the membrane potential histograms, computed after removing the spikes from the raw traces (Azouz and Gray 2000), is affected 


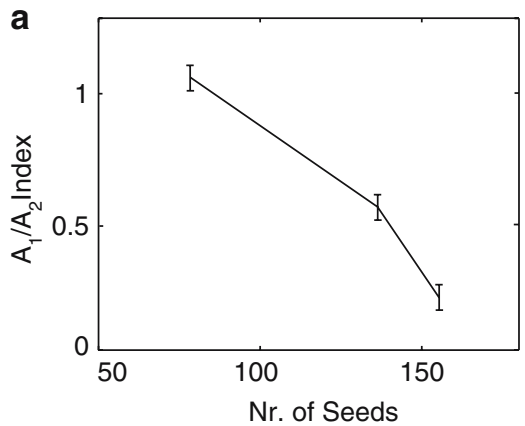

Fig. 5 (a) Quantifying the change in shape observed in the membrane potential histograms, as shown in Fig. 4c-d: the ratio of the more depolarized peak over the less depolarized one is plotted against different seeds frequencies; the resulting curve is a monotonic decreasing function of the frequency of the unitary

in the corresponding polarization states, then the A1/ A2-index is capturing this shift in the subthreshold dynamic. This observation is in good agreement with the change in bimodality for the presynaptic activity as described before. From the previous considerations, and as mentioned in section 3.1, increasing rates of unitary events are intuitively expected to increase output firing rates as well.

In the frequency domain, we analyzed the membrane-potential power spectra in the broad band between $5 \mathrm{~Hz}$ to $100 \mathrm{~Hz}$. We could not find any significant effect due to changes in the dynamics of highest-order correlation events. This confirms that stochastic resonance phenomena are not "boosting" any specific frequency component when $f_{\text {Sd }}$ changes.

\section{Supra-threshold properties.}

When analyzing the supra-threshold responses, we found no dependence of the coefficient of variation of the output inter-spike-intervals (ISIs) on the input frequency of the unitary events. The output coefficient of variation is constant, assuming a value close to 1 (Dean 1981).

Importantly, when looking at the output rates, which are strongly affected by changes of the frequency of unitary events. As shown in Fig. 5b, the output rates increase by more than $20 \%$ when the frequency of the unitary events is increased from $81 \mathrm{~Hz}$ to $153 \mathrm{~Hz}$. This again fits the previous intuitive explanation: when the number of unitary events in the input dynamics increases, the amplitude of the peaks in the PSTHs associated to the unitary events decreases, since the PSTH integral (the overall amount of spikes produced) is constant. However,

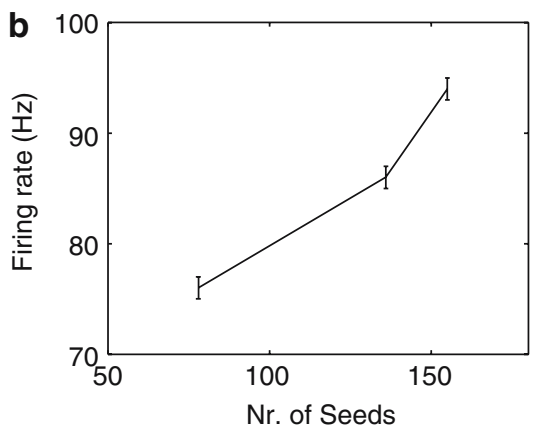

events. (b) For the passive model of a layer 5 pyramidal cell, the output firing rate is an increasing monotonic function of the frequency of the unitary events; the second order correlation properties (mean input firing rate, $\mathrm{CV}$ and pairwise correlation strength) are fixed for all the frequencies (see main text)

their impact on the target cell is still significant enough to elicit spikes. Thus, under these simulation conditions, higher rates of unitary events imply higher output firing rates as well.

\section{Rate properties during sub-optimal stimulation}

Given the above considerations, we can now formulate a model prediction. Suppose that the dynamics of the input network to a target neuron is characterized by fixed pairwise correlation strength, mean firing rate and frequency of the unitary events. Let's assume that the efficacy of the higher-order correlation events in eliciting postsynaptic spikes is very "low". This means that the peaks observed in the presynaptic population PSTH induce depolarized states in the postsynaptic membrane potential that can barely reach the threshold to produce output spikes. In other words, the probability of firing, given a presynaptic higher-order correlation event, should be roughly around $50 \%$.

Let's assume that this scenario is related to an input network that sub-optimally stimulates the target cell, i.e. an input network with low firing rates targeting a cell which responds accordingly with a low output rate. Suppose also that the frequency of the unitary events is not in the "saturation" limit (as explained above, Section 2.3). Thus it is still possible to increase the frequency of the unitary events while keeping the pairwise correlation strength constant. By increasing the frequency of unitary events, their amplitudes decrease even more, thus failing to elicit postsynaptic spikes. The prediction is that the output firing rate of the target cell should decrease.

We simulated such input dynamics for the passive layer 5 pyramidal cell (see Appendix) and, as shown in 
Fig. 6 (a-c) Left column, Output Seeds Triggered Analysis. This is a reverse correlation analysis to determine the temporal relationship between the seeds in the input activity, and the output spiking activity.

The plot shows the total number of spikes occurring in a time interval centered on the time of occurrence of the seeds, normalized by the total number of spikes in the spike train. Each plot refers to a different frequency of the seeds (insets): presynaptic seeds reliably trigger postsynaptic spikes. The right panels show the Input Seeds Triggered Analysis, where the same procedure as before is applied to the input activity profile (population PSTH): sharp, high-amplitude barrages of presynaptic inputs are associated to the occurrence of a seed
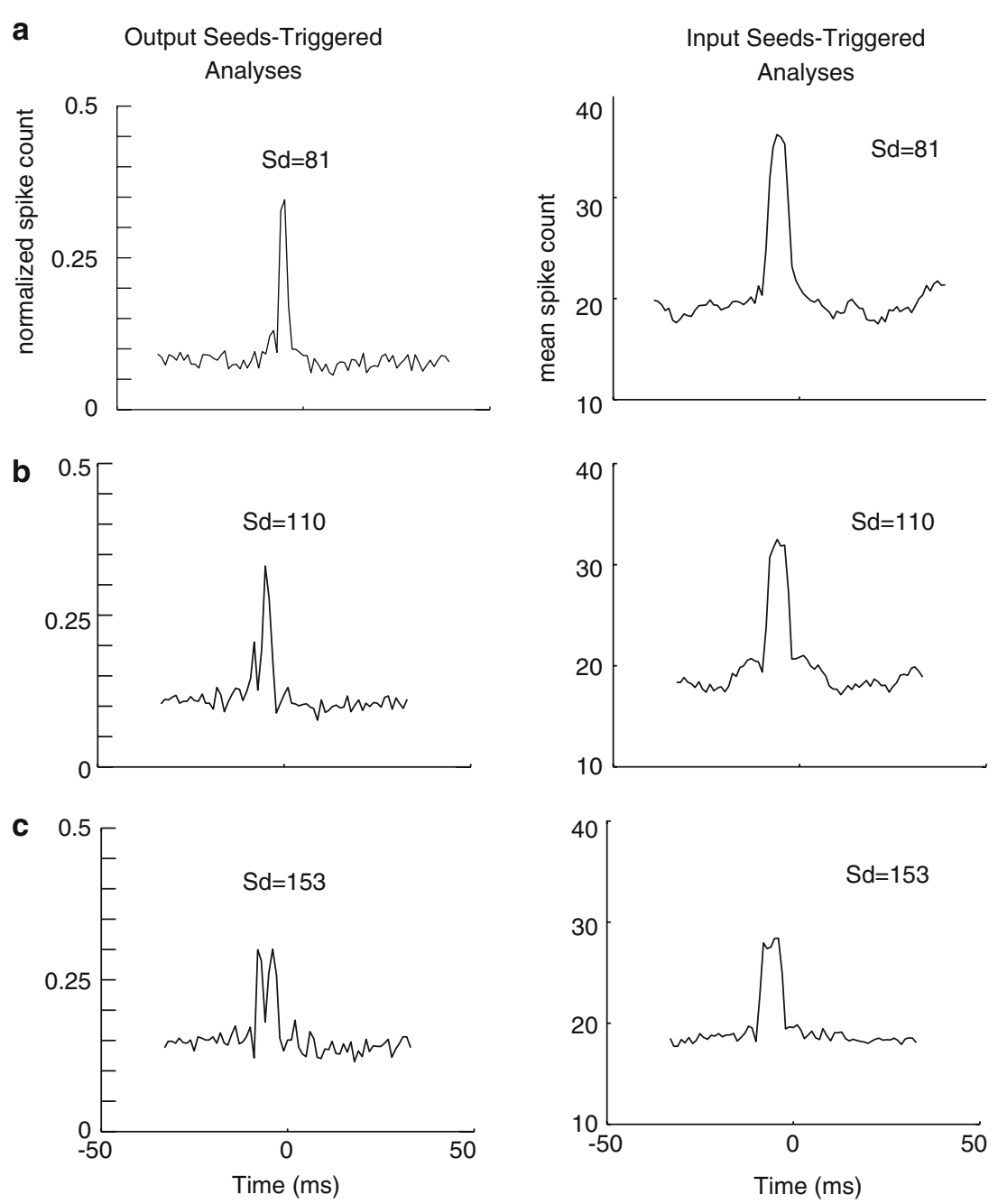

Fig. 7a, we found the expected decrease in the firing rate. With a frequency of the unitary events set to $81 \mathrm{~Hz}$ the output firing rate was $14 \mathrm{~Hz}$, and it kept on decreasing to $7 \mathrm{~Hz}$ when the frequency of unitary events increased to $153 \mathrm{~Hz}$. Thus, doubling the frequency of the unitary events halved the output rates. This effect is precisely opposite to what found for an optimally stimulated cell, as described before. The main message is that, when the frequency of the unitary events is increased, cells that are optimally stimulated tend to increase their firing rate, while cells that are sub-optimally stimulated behave in the opposite way. We stress again that this result is solely due to variations in the dynamics of unitary events, while leaving all the second order correlation properties unchanged.

\section{Impact on spike timing}

In order to analyze the impact of the unitary events on the output spike timing, we computed the conditional probability, $P(\mathrm{Sp} \mid \mathrm{Sd})$, that, given a unitary event (or a seed, 'Sd') in the presynaptic activity, the target cell would produce an output spike ('Sp'). Seeds-triggered correlation analysis can numerically approximate the conditional probability. If a seed occurs at time $t_{0}$, we looked at the spiking activity at the output stage using a time window centered around $t_{0}$ and having a width $T_{\mathrm{w}}=3 \times$ ISI (Inter-Spike Interval). The use of a variable duration for $T_{\mathrm{w}}$ has the advantage to make the conditional probability independent from the specific firing rate in the window of analysis. Results are shown in Fig. 6a-c, left column. To study how the algorithm was generating unitary events in relation to the seeds, we also computed the average network activity centered around the time of occurrence of a seed, and we refer to this measure as the input seeds-triggered analysis, as opposed to the $P(\mathrm{Sp} \mid \mathrm{Sd})$, which we will refer to as the output seeds-triggered analysis. The input seeds-triggered analysis is essentially the same correlation analysis as the output seeds-triggered analysis, where the population PSTH is used instead of 
the output spike train (Fig. 6a-c, right panels). These results show that the presence of a unitary event in the population activity is, as expected, time-locked to the seeds and triggers spikes on the postsynaptic cell. Often the cell spikes more than once (burst) and this accounts for the double peak that appears at a time-lag of roughly $2 \mathrm{~ms}$ from the first one (refractoriness). The width of the peaks observed in the input seeds-trig gered analysis is indeed comparable to the width of the cross-correlograms, as explained before (compare, for example, Fig. 6a, right panel, with Fig. 2e, right panel) (Bothé et al. 2000). Complementary to the output seeds-triggered analysis, we computed the spike-triggered input analysis, which is a reverse correlation analysis to reveal the presynaptic events that are time related to the output spikes. As expected, on average, output spikes are preceded by higher-order correlation events, Fig. 7b. A consequence of this observation is that the coefficient of variation of the presynaptic inter-unitary-events intervals strongly affects the coefficient of variation of the output inter-spike intervals.
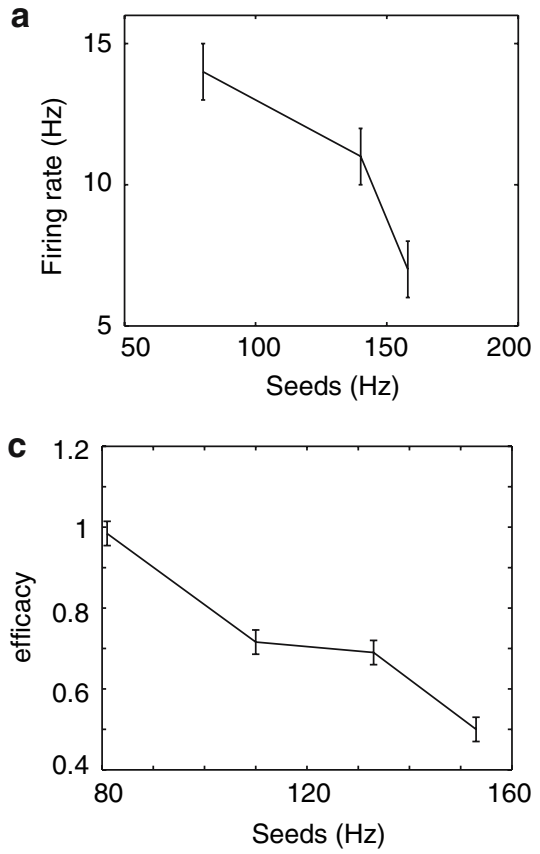

Fig. 7 (a) Dependence of the output firing rate of a layer 5 pyramidal cell on the seeds frequency, during sub-optimal stimulation. When the frequency of the unitary events increases, the output rate decreases. This behavior is opposite to what observed during optimal stimulation, as shown in Fig. 5b. (b) Spikes Triggered Input Analysis. This is a reverse correlation analysis computed by averaging the population PSTH in a time interval centered on the time of occurrence of the output spikes. The result emphasizes the presence of a higher-order correlation event in the input whenever an output spike is produced. (c) Efficacy of the seeds in eliciting spikes. This parameter has been
Essentially, the time variability of the unitary events in the input network constrains the variability of the spike timings of the output train. This result is in good agreement with previous experimental studies (Stevens and Zador 1998).

To characterize the variability of the conditional probability, $P(\mathrm{Sp} \mid \mathrm{Sd})$, while changing the frequency of the unitary events, we introduced a measure of the efficacy of the unitary events in eliciting postsynaptic spikes, defined as the area of the central peak of the output seeds-triggered function, divided by the offset level (the area of the curve under the peak, cutting at the baseline level). This dimensionless index can assume values that are greater than or equal to zero. If zero, i.e. a flat curve the postsynaptic spiking activity is completely unrelated to the seed timings, while greater than zero, implies increasing temporal dependence between output spikes and unitary events. As shown in Fig. 7c, this parameter is a decreasing function of the number of unitary events. Since the mean population firing rate is kept constant, when the frequency of the
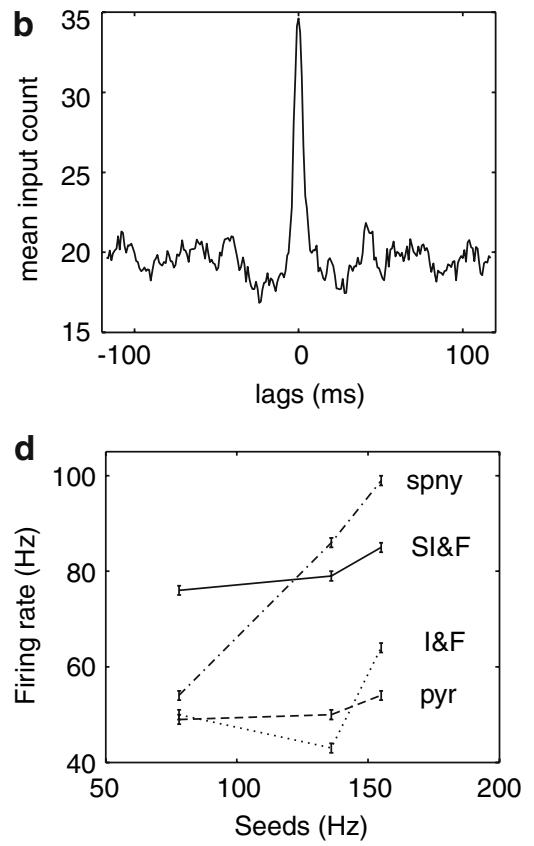

derived from the output seeds-triggered analysis as the ratio of the area of the central peak over the area of the plot below the peak (see Method section). An additional data point at $110 \mathrm{~Hz}$ has been used to better evaluate the efficacy profile. (d) Seedsto-rate transfer functions for different modeled cells: "spny" refers to the layer four spiny stellate cell, SI\&F refers to the integrate and fire neuron with stretched EPSPs (see main text), I\&F, to the standard Integrate and Fire model, "pyr" to the layer 5 pyramidal cell with voltage dependent mechanisms (the passive case is shown in Fig. 5b) 
unitary events is increased, their amplitude must decrease, thus their efficacy in eliciting postsynaptic spikes decreases.

The results of Section 3 can be summarized as follows: the overall output rate is determined by the balance between two opposite factors: on one side, the efficacy of the unitary events, which decreases with increasing rates of unitary events, causing a decrease of the output firing rates accordingly. On the other side, the rate of the unitary events, which tends to increase the output firing rates. The mean activity level of the presynaptic network determines which of the two factors will win: when the mean firing rate of the population activity is "high" $(>30 \mathrm{~Hz})$, the latter factor is dominant, and an increase of output rates is observed with increasing rates of unitary events. In this case, the decrease of the efficacy is not strong enough to silence the output activity, and the unitary events keep on triggering postsynaptic spikes. However, when the mean input rates are low $(<30 \mathrm{~Hz})$, the decrease of the efficacy is much more dramatic: the unitary events can still affect the subthreshold dynamics, but they are not able to trigger postsynaptic spikes.

\section{Controls: active mechanisms and different morphologies}

The analyses shown so far have been performed using a passive model of a layer five pyramidal cell. As a control, we tested the model with different cell morphologies and a number of voltage dependent mechanisms during optimal stimulation.

On a first set of experiments we used a morphologically reconstructed layer 4 spiny stellate cell, inserting non-inactivating $\mathrm{Na}^{+}$channels, high-threshold $\mathrm{Ca}^{2+}$ channels, $\mathrm{Ca}^{2+}$-dependent $\mathrm{K}^{+}$channels, muscarinic $\mathrm{K}^{+}$channels $\left(\mathrm{I}_{\mathrm{M}}\right)$ and transient $\mathrm{K}^{+}$channels $\left(\mathrm{I}_{\mathrm{A}}\right)$ in the dendrites (Mainen and Sejnowski 1999) (see Appendix for the parameters used). We then repeated the simulations using exactly the same input statistics. The results show that the unitary events-to-rate transfer function still exhibits the same qualitative behavior as observed for the passive model, i.e. the rate is a monotonically increasing function of the frequency of the unitary events, Fig. 7d. We then inserted the previously described voltage dependent mechanisms also in the layer five pyramidal cell, adding an anomalous rectifying current $I_{h}$, with a positive gradient for the peak conductance going from layer four to the apical dendrites in layer one, as reported experimentally (Berger et al. 2001). As shown in Fig. 7d, the transfer function still retained the same qualitative features, having a monotonically increasing behavior. However, such monotonic behavior disappeared as soon as we got rid of the morphology, simply by redirecting the same inputs to a conductance based integrate and fire neuron (see Appendix for parameters), Fig. 7d. In order to narrow down which mechanism was responsible for the observed change in the transfer function, we performed a sequence of controls by selectively morphing different dendritic parts into an equivalent single compartment, first the whole basal morphology, then the apical one, and finally by morphing the whole cell into a 3-compartment model (Destexhe 2001). The results show that the intact morphology of the basal dendrites is a necessary requirement for the emergence of a monotonic behavior. Note that each unitary events-to-rate transfer function in Fig. 7d, spans different rate intervals. This is simply due to the fact that since every modeled cell has different electrotonic properties, we adjusted the passive parameters in order to keep the output rate within the $40 \mathrm{~Hz}$ to $100 \mathrm{~Hz}$ frequency band, meant here as a "consistency range" of output frequencies with respect to the mean input firing rates.

One possible explanation for the observed change in the monotonic behavior is related to the filtering properties of the basal dendrites: when targeted by a unitary event, these "thin long cables" become isopotential compartments. Such a quick potential change occurs in parallel across all the basal dendrites involved, and its duration is "stretched" in time due to the passive cable properties of the dendrites (Koch 1999). The overall effect is a sustained current injection into the soma with a very fast onset. This phenomenon cannot be implemented in a single geometrical point, i.e. an integrate and fire neuron. We tested the hypothesis that the disappearance of the monotonic behavior was related to an electrotonic transformation operated by the basal dendrites by prolonging the time duration of the EPSPs, i.e. by increasing the durations of the time constants in the mechanisms controlling the AMPA synapses (and modifying the GABA time constants accordingly to keep the balance of the cell fixed), while leaving the presynaptic dynamics unchanged. The response properties of such a modified integrate and fire neuron switched again from convex to monotonic, Fig. 7d.

We also repeated the analyses done in Section 3.1.3 for the input seeds-triggered analysis, output seedstriggered analysis, and spikes-triggered input analysis, with voltage dependent mechanisms and different morphologies (data not shown). We found the same qualitative results as for the passive model of the layer five pyramidal cell. Active mechanisms and different morphologies produce only quantitative differences, in 
a very similar way as for the output rates shown in Fig. 7d. Similarly, in low input regimes, we found that active mechanisms and changes in the synaptic properties do not affect the decreasing monotonic behavior of the seeds-to-rate transfer function.

Finally, we ran controls for the balance of the cells and for the tuning properties of the inputs: unbalancing the cell by increasing the strength of the AMPA synapses (or decreasing the strength of the GABA synapses) shifted the curves shown in Fig. 7d upwards; while unbalancing the cell by increasing the strength of the GABA synapses (or decreasing the strength of the AMPA synapses) produced the opposite effect. Instead, a change in the slope of the seeds-to-rate transfer functions is observed whenever voltage dependent mechanisms that are related to dendritic boosting of the backpropagating depolarizing potentials, such as sodium and calcium channels, are modified. Also changes in $\mathrm{I}_{h}$ conductances significantly affect the slopes of the transfer functions. Nonetheless, the magnitude of these effects is such that the monotonic behavior of the seeds-to-rate transfer functions is preserved.

These controls lead to two considerations: first, active mechanisms in the neuron modify the quantitative, but not qualitative behavior of the unitary events-to-rate transfer function. This is true for voltage dependent mechanisms having both short (few milliseconds) and long lasting (hundreds of milliseconds) time constants. Second, unitary events can have different effects depending on the morphology of the target cell. The basal morphology seems to play a crucial role and the temporal filtering properties of the basal dendrites are a necessary ingredient for the emergence of the described phenomena.

\section{Linking higher-order correlations to sparseness and neuronal selectivity}

In the following we speculate on how the differential impact of higher-order correlation events on the output response properties of modeled cortical neurons could help to understand well described experimental phenomena traditionally not related to spike correlations.

\section{Population sparseness}

To quantify the impact of the unitary events' variability on the overall presynaptic population dynamics, we studied the dependence of population sparseness on the frequency of the seeds. This measure of population variability is often related to electrophysiological studies showing that stimulation of the non-classical receptive field ( $\mathrm{nCRF}$ ) of a cells induces decorrelations in the activity of neurons having different stimulus selectivity (Vinje and Gallant 2000). The quantitative measure of this effect is related to an increase in sparseness, which is a measure of the way information is distributed among spikes within and between spike trains (Vinje and Gallant 2000). Thus, following nonclassical receptive field stimulations, it has been shown that moderate neuronal responses tend to disappear and the proportion of small and large responses increases, thus increasing sparseness as well (Vinje and Gallant 2000; Willmore and Tolhurst 2001).

Given that such results closely resemble the differential effect observed in our simulation, we computed the time course of population sparseness in the presynaptic spike trains. We binned every input spike train using a $30 \mathrm{~ms}$ time window and we interpreted the corresponding spiking activity as the response to a "virtual" external stimulation. Then, for every time bin, we computed the population sparseness using its standard definition $K_{\mathrm{p}}=\left\{\frac{1}{N} \sum_{j=1}^{N}\left[\frac{r-\bar{r}}{{ }^{4}}\right]^{4}\right\}-3$ (i.e. the Kurtosis of the response distribution) with $\bar{r}$ and $\sigma_{r}$ the mean and standard deviation of the responses across
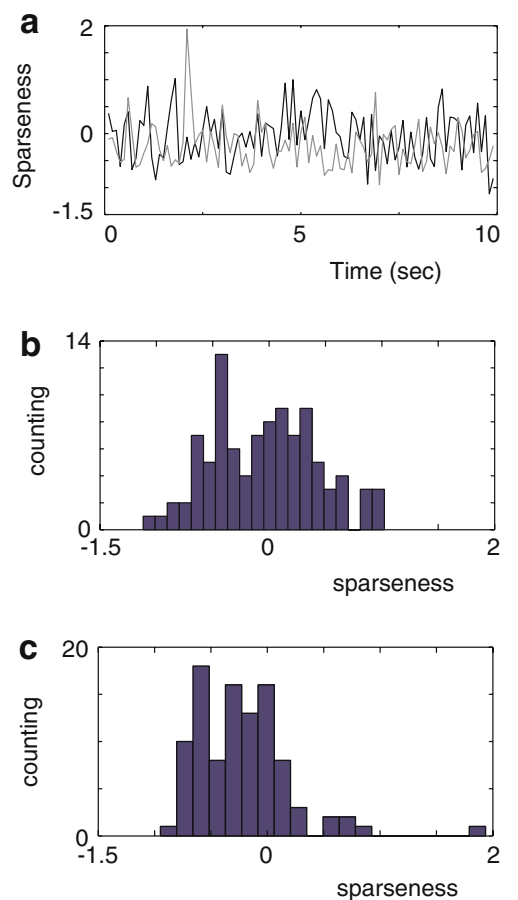

Fig. 8 (a) Time evolution of the population sparseness: the light gray and black traces refer to seeds frequencies of $153 \mathrm{~Hz}$ and $81 \mathrm{~Hz}$, respectively. Despite the high variability throughout the whole simulation time $\mathrm{T}$, histograms of the sparseness traces (b and $\mathbf{c}$ panel for $81 \mathrm{~Hz}$ and $153 \mathrm{~Hz}$, respectively) show a shift in the mean of the distributions toward lower values for increasing frequencies of the unitary events 
the population of neurons. When the frequency of the unitary events is increased, sparseness assumes more negative values (Fig. 8a-c).

If, for example, one considers an input and output network, increasing the rate of higher-order correlations in the input network causes two opposite effects: (1) a decrease of sparseness in the input network, as shown above. (2) Since in the output network moderate responses tend to disappear and the proportion of small and large responses increases, then sparseness increases in the output network.

Thus, changes in population sparseness can be explained within the general framework of the dynamics of the higher-order correlation events. Standard second order correlation properties are fixed, and effects on population sparseness are observed, solely due to variations in the higher-order correlation properties of the network dynamics.

\section{Feature selectivity}

Another issue related to the differential impact of higher-order correlation events, is the feature-selectivity of cortical neurons. If an increase in the frequency of the unitary events enhances the firing rate of optimally stimulated cells, while decreases the responses to sub-optimally stimulated neurons, then the selectivity (tuning properties) of the neurons should also be affected.

We used the data introduced in Section 3.2.2 and 3.2.3, to quantify this idea. Using a standard orientation tuning curve (Ferster and Miller 2000), with a peak firing rate at $90^{\circ}$, we produced changes in the frequency of the unitary events. We then analyzed the impact on the firing rates as done for Fig. $5 b$ and Fig. 7a, for the optimal and sub-optimal responses. At $80^{\circ}$, when the unitary events frequency increased from 81 to $153 \mathrm{~Hz}$, the firing rate of the modeled neuron increased by the $27 \%$ (from $75 \mathrm{~Hz}$ to $95 \mathrm{~Hz}$ ), while at $57^{\circ}$ it decreased by the $50 \%$ (from $14 \mathrm{~Hz}$ to $7 \mathrm{~Hz}$ ). Accordingly, we computed the new tuning curve: a significant increase of the peak amplitude (with no significant decrease in the tuning width) and a significant decrease of the orthogonal responses are observed.

The phenomenon described could give insights about the mechanisms shaping the dynamics of the neuronal selectivity. For example, it is still hotly debated whether the orientation selectivity of visual neurons is mostly shaped by intracortical/recurrent mechanisms or by feed forward ones. Accordingly the neuronal selectivity is expected to change over time or remain stable during the time course of the response.
Experimental results have provided controversial evidences, but changes in the shape of the tuning curves have been reported both for cats and monkeys (for reviews see Shapley et al. 2003; Ferster and Miller 2000).

Here we suggest a mechanism that could explain some of the observed changes in neuronal selectivity. If, for example, circular statistic (i.e. circular variance) is used to quantify the feature selectivity of the neuron (Ringach et al. 2003), then the increased frequency of unitary events leads to an increase in neuronal selectivity. The orientation tuning properties of visual neurons could change during the time course of the response simply following the variability of higherorder correlation events in the input network, without any detectable change in the mean input firing rate, $\mathrm{CV}$, or pairwise correlation strength.

The above speculations are not meant to be formal proofs. Though, we have introduced these qualitative arguments to demonstrate the generality of the main findings, and the possibility to bridge traditionally unrelated aspects of the neuronal dynamics, such as unitary events, sparseness, and neuronal selectivity.

\section{Discussion}

In this study we have made a formal, systematic distinction between second order and higher-order correlation properties of the network dynamics, and we investigated whether neurons could detect changes produced solely in the higher-order correlation terms of the presynaptic dynamics. Higher-order correlations have been frequently discussed in the coding literature as potential carriers of information in synchronized cortical networks. Though, to what extent, if at all, neurons are sensitive to changes happening only in the higher order correlation statistics has not been thoroughly investigated. Indeed, the large majority of simulation studies dealing with correlations have investigated the impact of pairwise correlations in the input network on the response properties of target cells (typically point neurons), without considering the dynamics of higher-order correlation events (for a review see Bair 1999; Salinas and Sejnowski 2001). Second order and higher order correlation properties are two potentially independent sources of variability (Caianiello and Grimson 1975), which can contribute in parallel with different weights to the neuronal response properties, to an extent that has not yet been quantified. We have addressed this problem by fixing the second order correlation properties, and by allowing only changes in the higher-order correlation terms of the dynamics. The 
results found point to two conclusions: (1) higher-order correlation statistics of the input trains affect the temporal variability of the membrane potential, but not its mean value. (2) This phenomenon, combined with the spike non-linearity of neurons, explains why changes done only in the higher-order correlation statistics can cause a neuron to fire more or less depending on the mean input rate of the surrounding network. Moreover, we studied how the cell's morphology and active mechanisms (voltage dependent channels) affect the neuronal sensitivity to higher-order correlation events. We found that the temporal filtering properties of the basal dendritic tree play a key role in establishing such sensitivity.

\section{Correlation studies}

The idea that weak pairwise correlations can affect the neuronal response properties is a well studied phenomenon. Simulation studies have shown that the impact of weak pairwise correlations on the output response properties of an integrate and fire neuron depends on the balance of the neuron (Salinas and Sejnowski 2000). In a balanced regime, weak pairwise correlations affect the gain of the output and the firing is very irregular (high coefficient of variations). Other simulation studies with integrate and fire neurons have shown that weak pairwise correlations are necessary to account for the high spike timing variability observed in vivo, even for values of the balance shifted toward inhibition (Feng and Brown 2000). Similar conclusions have been reached using more sophisticated neuronal models with detailed morphological features, showing that dendritic non-linearities together with strong synchronized synaptic inputs can explain the highly irregular firing of cortical neurons (Softky and Koch 1993). Also in vitro studies have emphasized that highly synchronous presynaptic activity can play a critical role in explaining why cortical neurons respond very irregularly even with constant sensory stimulation or constant current injections (Stevens and Zador 1998). In agreement with these studies, our results support the idea that higher-order correlations in the input stage modulate the response properties of cortical neurons.

Nevertheless, a major difference in respect to previous studies is that we fixed all the degrees of freedom typically left free to vary such as the cell-balance, mean input firing rates, and pairwise correlations, narrowing down the impact of higher-order correlation statistics. Thus, the scope of this study, although overlapping in part with previous investigations, should be considered complementary to the above mentioned lines of research on weak pairwise correlations.
Tuning the simulation parameters

A number of assumptions have been made for the NEURON simulations. When dealing with detailed compartmental modeling of cortical cells, it is always possible to keep on adding neuronal mechanisms to implement more realistic modeled neurons. Moreover, it is never really clear which set of parameters should be preferentially chosen since, given the extremely higher-dimensionality of the parameter space, several different combinations of parameter sets can produce the same results. In this study the parameters controlling passive and voltage dependent mechanisms have been chosen according to a "perturbative approach" so that the qualitative features of the neuronal dynamics (output rates, spiking variability, seeds-to-rate transfer functions) were stable in respect to small changes in the parameter values.

Presynaptic dynamics: scope and limitations

When considering the presynaptic dynamics, the analysis depends on the assumptions made. An important one is the existence of higher-order correlation events.

Looking at studies dealing with correlations between large groups of neurons $\left(10^{5}\right.$ as an order of magnitude), experimental knowledge is available only at the time scale of hundreds of milliseconds, by using, for example, optical imaging, fMRI or EEG. On a faster time scale $(<10 \mathrm{~ms})$, the scale of interest when studying higher-order correlation events, no direct experimental knowledge is available. Current measurements can allow the simultaneous multi-electrode recordings of the spiking activity of cortical neurons that do not exceed an order of magnitude of $10^{2}$ neurons (Hoffman and McNaughton 2002). The problem can be rephrased saying that we have only indirect experimental hints about the statistical properties of the fast variability in the population dynamics.

More encouraging results come from theoretical/ computational studies, dealing with large-scale neural network simulations, which have shown that higher-order correlation events are a natural emerging property of the network's dynamics (Amit and Brunel 1997a; Brunel and Hakim 1999; Amit and Brunel 1997b). Theoretical investigations have shown that unitary events can degrade the temporal accuracy of a coding mechanism based on rate (Mazurek and Shadlen 2002), they have been claimed as necessary to explain the reliability of the spike timing (Mainen and Sejnowski 1995), and to generate high coefficients of variation, as generally observed in cortical spike trains (Salinas and Sejnowski 2000). The computational 
importance of higher-order correlation events becomes even more striking when considering coding mechanisms based on correlations, such as the theory of "binding by synchronization" (Von der Malsburg 1981), or dynamical models of information processing, such as "synfire chains" (Abeles 1991).

Finally, another unresolved issue, is the lack of experimental knowledge to understand what mechanisms could cause the simulated changes in the dynamics of the higher-order correlation events in a cortical network. This is a consequence of the limitations discussed before: if higher-order correlation events are difficult to detect, it is even more difficult to detect changes in their dynamics. On a positive side, theoretical studies have shown that several different mechanisms, such as, for example, synaptic depression (Loebel and Tsodyks 2002), neuromodulatory effects (Verschure and König 1999), and transmission delays (Horn and Opher 1999), could all contribute to induce significant changes in the higher-order correlation properties of the network dynamics.

\section{Coding}

The results found argue in favor of an important role played by higher-order correlation events in the neuronal coding mechanism. Indeed, following the well accepted idea that spike rates and spike timings are relevant coding variables (whatever the coding mechanism is), we showed that they are both significantly affected by dynamical changes of higher-order correlation events. In other words, higher-order correlation events cannot be considered as small perturbations or noise that can be averaged out by the ensemble's activity. Instead, they represent a dynamical phenomenon with a significant impact on "classical" coding variables. Thus, they should be considered key information carriers in cortical coding mechanisms based on rates or temporal correlations.

Acknowledgements This work was supported ETH-Zürich grant, the EU IST-2000-28127/BBW 01.0208 grant, the Swiss National Fund (SNF - grant No: 31-61415.01), and supported by James S McDonnell Foundation.

\section{Appendix}

The parameters used in the NEURON simulations are similar to the set of parameters used in a number of other modeling studies (for a review, see Mainen and Sejnowski 1999). The cell morphologies are available thanks to the kind concession of John Anderson, Tom Binzegger, and Kevan Martin. The passive properties for the layer five pyramidal and layer four spiny stellate cell (from cat, area 17) are the same: $C_{\mathrm{m}}=1 \mu \mathrm{F}$, $G_{\mathrm{m}}=0.07 \mathrm{mS} / \mathrm{cm}^{2}, \quad E_{\text {rest }}=-65 \mathrm{mV}, \quad R_{i}=90 \Omega \mathrm{cm}$, $T=37^{\circ} \mathrm{C}$. Standard Hodgkin and Huxley mechanisms have been inserted in the soma for generating spikes. We used several voltage dependent mechanisms (the mod files are freely available from the official NEURON website http://www.neuron.yale.edu/neuron/): non-inactivating sodium channels, $I_{\text {na }}$, with $E_{\mathrm{rev}}=50 \mathrm{mV}$ and $\bar{G}=500$ and $10 \mathrm{mS} / \mathrm{cm}^{2}$ in the soma and dendrites, respectively. $I_{\mathrm{L}}$, in the apical dendrites, $E_{\mathrm{rev}}=115 \mathrm{mV}$ and $\bar{G}=5.1 \mathrm{mS} / \mathrm{cm}^{2} . I_{\mathrm{k}}$ in the soma, $E_{\mathrm{rev}}=-77 \mathrm{mV}$ and $\bar{G}=50 \mathrm{mS} / \mathrm{cm}^{2}$. Calcium dependent potassium channels, $I_{\mathrm{C}}, E_{\mathrm{rev}}=-77 \mathrm{mV}$ and $\bar{G}=30 \mathrm{mS} / \mathrm{cm}^{2}$, in the apical dendrites. Dendritic and somatic $I_{\mathrm{A}}, \quad E_{\mathrm{rev}}=-66 \mathrm{mV}$ and $\bar{G}=10 \mathrm{mS} / \mathrm{cm}^{2}$. Dendritic $I_{\mathrm{M}}, E_{\mathrm{rev}}=-77 \mathrm{mV}$ and $\bar{G}=30 \mathrm{mS} / \mathrm{cm}^{2}$ and finally, only for the layer five pyramidal cell, we used $I_{\mathrm{h}}$ current, with layer-dependent conductances (Berger et al. 2001): $E_{\mathrm{rev}}=-50$, with $\bar{G}=32,22,7,2.5 \mathrm{mS} /$ $\mathrm{cm}^{2}$ going from layer 1 to layer 4 respectively. The conductance-based integrate and fire neuron (I\&F) consists of an RC circuit with a $g_{\text {leakage }}$ and $\mathrm{H} \& \mathrm{H}$ mechanisms in the soma to make the cell fire. For the synaptic activations, we used standard alpha synapses, both for excitations (AMPA) and inhibitions (GABA). $E_{\mathrm{rev}}=0 \mathrm{mV}, \tau_{1}=0.3 \mathrm{~ms}, \tau_{2}=4 \mathrm{~ms}$ for the AMPA, and $E_{\mathrm{rev}}=-70,-95 \mathrm{mV}, \tau_{1}=4.5,35 \mathrm{~ms}, \tau_{2}=7$, $40 \mathrm{~ms}$ for the GABA-A,B, respectively. The specific equations controlling the time course of the synaptic activations are as in Mainen and Sejnowski (1999). We inserted 500 GABA-A and 500 GABA-B synapses distributed randomly in the basal dendrite and initial part of the apical trunk. The associated input spike trains follow the same correlation structure as for the 4000 AMPA, which instead are randomly distributed all over the dendritic arborisations. To provide suboptimal stimulation to the modeled neurons

\section{References}

Abeles M (1991) Corticonics. Cambridge Univ Press

Amit DJ, Brunel N (1997a) Dynamics of a recurrent network of spiking neurons before and following learning. Network 8:373-404

Amit DJ, Brunel N (1997b) Model of global spontaneous activity and local structured activity during delay periods in the cerebral cortex. Cerebral Cortex 237-252

Azouz R, Gray CM (2000) Dynamic spike threshold reveals a mechanism for synaptic coincidence detection in cortical neurons in vivo. PNAS 97:8110-8115

Bair W (1999) Spike timing in the mammalian visual system. Curr Opin Neurobiol. 9(4):447-453

Berger T, Larkum ME, Luscher HR (2001) High I(h) channel density in the distal apical dendrite of layer $\mathrm{V}$ pyramidal 
cells increases bidirectional attenuation of EPSPs. J Neurophysiol 85:855-868

Bernander O, Kock C, Douglas RJ (1994) Amplification and linearization of distal synaptic input to cortical pyramidal cells. J Neurophysiol 72(6):2743-2753

Bothé SM, Spekreijse H, Roelfsema PR (2000) The effect of pair-wise and high-order correlations on the firing rate of a post-synaptic neuron. Neural Computation 12:153-179

Brunel N, Hakim V (1999) Fast global oscillations in networks of integrate-and-fire neurons with low firing rates. Neural Computation 11:1621-1671

Caianiello ER, Grimson WE (1975) Synthesis of boolean nets and time behavior of a general mathematical neuron. Biol Cybern 18:111-117

Dean AF (1981) The variability of discharge of simple cells in the cat striate cortex. Exp Brain Res 44:437-440

Destexhe A (2001) Simplified models of neocortical pyramidal cells preserving somatodendritic voltage attenuation. Neurocomputing 38-40:167-173

Engel AK, König P, Gray CM, Singer W (1990) Stimulusdependent neuronal oscillations in cat visual cortex: Intercolumnar interaction as determined by cross-correlation analysis. Eur J Neurosci 2:588-606

Feng J, Brown D (2000) Impact of correlated inputs on the output of the integrate and fire model. Neural Computation 12(3):671-692

Ferster D, Miller KD (2000) Neural mechanisms of orientation selectivity in the visual cortex. Annu Rev Neurosci 23:441-471

Gray CM, Viana di Prisco G (1997) Stimulus-dependent neuronal oscillations and local synchronization in striate cortex of the alert cat. J Neurosci 17:3239-3253

Grün S, Diesmann M, Aersten A (2001) Unitary events in multiple single-neuron spiking activity:I detection and significance. Neural Computation 14:43-80

Hines ML, Moore JW, Carnevale NT (2000) NEURON, Duke and Yale University

Hoffman KL, McNaughton BL (2002) Coordinated reactivation of distributed memory traces in primate neocortex. Science 297:2070-2073

Horn D and Opher I (1999) Collective excitation phenomena and their applications. In: Maass W (ed) Pulsed neural networks, Bishop CM MIT press, pp 297-320

Kisvarday ZF, Toth E, Rausch M, Eysel UT (1997) Orientationspecific relationship between populations of excitatory and inhibitory lateral connections in the visual cortex of the cat. Cerebral Cortex 7:605-618

Koch C (1999) Linear cable theory. In: Biophysics of computation, Anonymous. Oxford University Press, pp 25-48

Loebel A, Tsodyks M (2002) Computation by ensemble synchronization in recurrent networks with synaptic depression. J Comput Neurosci 13:111-124

Mainen ZF, Sejnowski TJ (1995) Reliability of spike timing in neocortical neurons. Science 268:1503-1506

Mainen ZF and Sejnowski TJ (1999) Modeling active dendrtitic processes in pyramidal neurons. In: Koch C, Segev I (eds) Methods in neuronal modeling. MIT press, pp 171-210

Mazurek ME and Shadlen MN (2002) Limits to the temporal fidelity of cortical spike rate signal. Nature neurosci 5:463-471

Ringach DL, Hawken MJ, Shapley R (2003) Dynamics of orientation tuning in macaque V1: the role of global and tuned suppressio. J Neurophysiol 90:342-352

Rudolph M and Destexhe A (2001) Correlation detection and resonance in neural systems with distributed noise sources. Phys Rev Lett 86(16):3662-3665

Salinas E, Sejnowski TJ (2000) Impact of Correlated Synaptic Input on Output Firing Rate and Variability in Simple Neuronal Models. J Neurosci 20:6193-6209

Salinas E, Sejnowski TJ (2001) Correlated neuronal activity and the flow of neural information. Nat Rev Neurosci 2:539-550

Shapley R, Hawken M, Ringach DL (2003) Dynamics of orientation selectivity in the primary visual cortex and the importance of cortical inhibition. Neuron 38:689-699

Softky WR, Koch C (1993) The highly irregular firing of cortical cells is inconsistent with temporal integration of random EPSPs. J Neurosci 13(1):334-350

Stevens CF, Zador AM (1998) Input synchrony and the irregular firing of cortical neurons. Nature neurosci 1:210-217

Verschure PF, Konig P (1999) On the role of biophysical properties of cortical neurons in binding and segmentation of visual scenes. Neural Comput 11:1113-1138

Vinje WE, Gallant JL (2000) Sparse coding and decorrelation in primary visual cortex during natural vision. Science 287:1273-1276

Von der Malsburg C (1981) The correlation theory of the brain function. Internal Report 81-2. Max-Plank-Institute fir Biophysical Chemestry, Göttingen

Willmore B, Tolhurst DJ (2001) Characterizing the sparseness of neural codes. Network 12:255-270 